\title{
An Agent-based Simulation System for Evolution of Interplay between E-Commerce Vendor and Consumers
}

\author{
Guoyin Jiang*, Xingjun Liu and Youtian Wang \\ School of Information Management, Hubei University of Economics, Wuhan, \\ 430205, China \\ jiangguoyin@hbue.edu.cn; Liuxingjun@tom.com; wangyoutian@ninul.com
}

\begin{abstract}
We use computational experiment method integrated multi-agent, advanced GA (Genetic Algorithm), and complex network to study the interactive evolution of e-commerce vendors and consumers. Key algorithms such as generating algorithm for consumer network, and advanced GA based on CA (Cellular Automata) for service vendor decisions, etc, are designed for the evolution behaviors of both e-commerce vendors and customers. We program the computational experiment system with NetLogo, and a simulation experiment is given. The experiment system can provide technique support to e-commerce firms in decision making with regards to service providing, consumer behavior management, etc.
\end{abstract}

Keywords: Advanced GA; e-commerce; Cellular Automata; Agent

\section{Introduction}

Along with the advanced network and information technology purse into the business application, diverse collaboration modes come into being into e-commerce; the depth collaboration or synergy has been the trend of the development of e-commerce. The depth collaboration is the fusion of different things into a harmony to share information and resources in order to achieve an overall performance that is better than the sum of all constituent parts otherwise [1], i.e. " $1+1>2$ ". For example, big e-commerce enterprises strengthen their search technology one after another to collaborate with search. In 2007, alibaba.com allied with yahoo, in October, 2010, taobao.com allied with Microsoft Bing.com to create etao.com, which is an independent search engine dedicated to taobao.com; in June, 2010, taobao.com and Yahoo Japan, which has Softbank Japan as the stakeholder, simultaneously put online Taobao Japan and Yahoo Japan's China Mall. In order to flex its value of brand, Tencent integrated QQ and Paipai such that Paipai can share QQ's user pool and QQ user can go online shopping by just one click on the dashboard. Tianya was also attempting to transfer their registered members to consumers of online market which is proved by Tianya.

Under the environment of collaborative e-commerce, service vendor will adjust its management policy in line with outside market and consumers, such as to adjust price, introduce new technology, build up brand, etc. Consumers will act accordingly in light of service vendors' change of management policy, to determine whether to buy or wait and see, or totally abandon the brand. Hence, service vendor and consume interact dynamically, and there decision making processes are interlaced and evolving together. Current literatures mainly concentrate on the solo evolution of e-commerce vendor, or the solo evolution of consumer decision making; research of the co-evolution is rare[2]. In

* Corresponding author. Guoyin Jiang

E-mail address: jiangguoyin@hbue.edu.cn Tel.: +86 2781972191 
this paper, we attempt to introduce computational experiment methods to represent the co-evolution scenario.

Computational experiment method is a comprehensively integrated method involving computation technology, artificial intelligence, complex system theory and evolution theory [3]; it can be used to visualize the relation between management scenario and microscopic subjects' behavior via computer. Use of computational method to study system evolutions will help reveal the deep rooted inner workings and microscopic laws of an evolutionary system; so this method is widely used in management and economics when it comes to complex behavior research. Traditional qualitative research can expose concept, framework and observations, while empirical research can analyze influencing factors and behavioral mechanisms on a static basis, that is, the analysis is about an event at a fixed time and a fixed venue; evolutionary game theory can analyze dynamic evolutionary process but with a coarse granularity, and the result is often macroscopic. Agent-based modeling or other computational methods regarding behavior research can study dynamic complex system with a more concrete and more microscopic result $[4,5]$.

Consumers are sited at a social network, they communicate with other by social media or online tools, for example blogs, Facebook and others. Prior empirical works examine the network structure, the findings indicate that, the www or web is a scale-free network $[6,7]$, the network has a small-world feature, where most users are not direct neighbors of one another, but they can be reached from a small number of hops or steps, and the network has a degree distribution following a power law $[6,8]$. In this work, we use scale-free network as a surrounding where agent can interact with others.

In this paper, we will discuss the implementation of such systems, including main algorithms, and fundamental principles of the interactive evolution between e-commerce service vendor and consumer groups under collaborative e-commerce circumstances.

\section{Mechanisms of the Interaction between Service Vendor and Consumer Behavior}

To cater to consumers' various demand and competitor's tense competition, collaborative service in e-commerce need to dynamically change management strategy; Generally price, technique, and brand strategy will improve through learning, bringing force new creative services.

For e-commerce oriented collaborative service, consumer not only experience shopping process themselves to feel the service capacity and quality in regards to the brand and technology alliance of e-commerce service vendors, but also heed the word of mouth from other related consumers, as well as the advertisement or publicity of mass media. Consumers form a special structured network among themselves. The consuming status, including buying $\backslash$ to buy $\backslash$ dormant, of individual consumer on the network nodes changes over time; the message from one consumer to another as well as the effect of mass media also change. All of these will change the status of consumers.

As market circumstances change, the network structure of consumer groups will transform dynamically and an interactive co-evolution between consumer group and service vendors are formed. That is, service vendors will make decision according to market circumstances, consumer network structures and consuming trends to implement different strategy in price $\backslash$ brand $\backslash$ technology innovation; on the other hand, facing the portfolio of service vendor measures, consumer will adopt different purchase strategy thus consumers' shopping status would transit from one to another. Consumer group and service vendors co-evolve over time and the process can be described in Figure 1. 


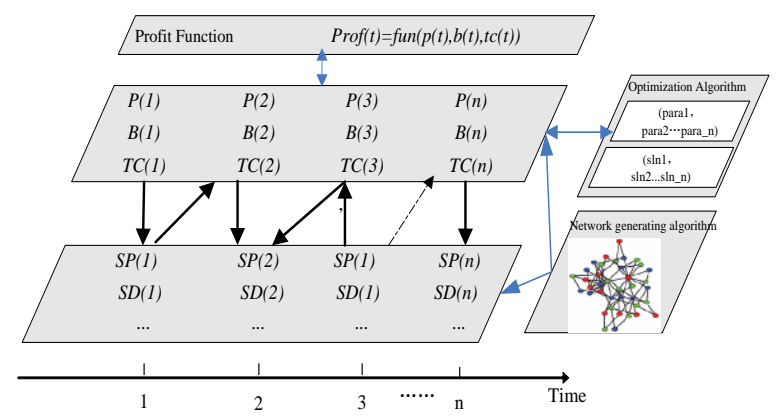

Figure 1. Mechanisms in the Interactive Co-evolution between Collaborative E-commerce Service Vendor and Consumer Group

In Figure 1, $P$ is price strength, $B$ is brand strength, $T C$ is technology strength, $S P$ is number of shopping persons, $S D$ is number of shopping dormant persons, Prof is time-varying profit model (in other words, service vendor's profit model under different $P, B$ and $T C)$. In our system, the profit model is:

$$
\begin{aligned}
& \text { prof }(t)=a \cdot\left(2-c \cdot(P(t)+1)^{\alpha} \cdot(B(t)+1)^{\beta} \cdot(T C(t)+1)^{\gamma}\right)- \\
& c_{1} \cdot P(t)-c_{2} \cdot B(t)-c_{3} \cdot T C(t) \\
& \text { s.t. }\left\{\begin{array}{l}
0 \leq P(t) \leq 1 \\
0 \leq B(t) \leq 1 \\
0 \leq T C(t) \leq 1
\end{array}\right.
\end{aligned}
$$

The model 1 is an optimal model at multi-periods, we cannot gain direct solution for it by traditional mathematic method, therefor, a optimal algorithm is used to find approximate solution for model 1, the method will be introduce in sub section 3.2.

\section{Key Algorithms for Interactive Evolution Experiment}

\subsection{Consumer Network Generation Algorithm}

Consumer network in our study is a scale-free network. The input of the network is denoted as $\operatorname{Input}\left(n_{0}, m\right)$, where $n_{0}$ is the initial cardinality of network nodes. $m(m \leq n)$ is the cardinality of a new node's connections when the new node is introduced into the network.

\section{Initial network generation algorithm}

a) Initialize network space

b) Call node generating algorithm, generating two nodes randomly in the space

c) Execute edge generating algorithm, connecting the initialized two nodes

d) Repeat the following operations till the cardinality of nodes collection satisfy preset conditions:

1) Generate a new node

2) Find nodes to be connected

3) Execute edge generating algorithm, connecting found nodes and new node.

4) Execute layout-spring algorithm in Netlogo system to layout these nodes

5) Return

Network nodes generating algorithm

a) Generate random coordinates: $(x, y)$

b) Execute drawing algorithm, drawing a circle with $(x, y)$ as the center.

c) Coloring the nodes

\section{Edge connecting algorithm}

a) Get the coordinates of two nodes 
b) Execute lining tool, drawing an edge between these two coordinates

Algorithm to find nodes to be connected

a) Declare var total, and assign a random number in the range $[0$, total number of edges]

b) Declare var partner to denote a node to be connected and initialize it to null

c) For each node currentNode:

1) Let $n c$ as the total number of connections

2) If partner=null, and $n c>$ total, then let partner=currentNode; or else let total=total $-n c$

3) Output partner

\subsection{Advanced GA based on Cellular Automata}

Classical GAs and adapted versions have been successfully applied to all kinds of fields [9-15]. In face of multi-cycle optimization problem, such algorithms will run for too long a time. In this study, we will try to improve classical GAs to run faster while retaining a relatively precise solution [4].

Cellular Automata can simulate emergence, that is, local movements will diffuse and bring about the macroscopic evolution of organisms. In this research, we will introduce cellular automata's local diffusion capability to implement the local movement of chromosome in GA. That is, each cell will stand for one chromosome, interacting will other chromosomes to disseminate information and optimize its behaviors.

All cells are placed in a lattice $L$, and each grid is a cell (in other words, chromosome). The size of $\mathrm{L}$, or the total number of cells, is $m^{*} n$, where $m, n \in$ Integer; $L$ denotes how large an evolving race is. Every cell can interact with its neighbors, the 8 cells around it as defined by Moore-neighborhood in Figure 2.

\begin{tabular}{|c|c|c|c|}
\hline $\mathrm{A}_{1,1}$ & $\mathrm{~A}_{1,2}$ & $\ldots$ & $\mathrm{A}_{1, \mathrm{n}}$ \\
\hline $\mathrm{A}_{2,1}$ & $\mathrm{~A}_{2,2}$ & $\ldots$ & $\mathrm{A}_{2, \mathrm{n}}$ \\
\hline$\cdots$ & $\ldots$ & $\ldots$ & $\ldots$ \\
\hline $\mathrm{A}_{\mathrm{m}, 1}$ & $\mathrm{~A}_{\mathrm{m}, 2}$ & $\ldots$ & $\mathrm{A}_{\mathrm{m}, \mathrm{n}}$ \\
\hline
\end{tabular}

Figure 2. Cells of CA

The optimization process of GA based on CA is the same as that of traditional GA, including phases like: selection, crossover, and mutation, but its interactions are restricted locally with the eight neighboring cells and the fittest cell will be selected to cross over, and the generated one will replace the central cell.

\section{Implementation of the System and Experiment}

\subsection{Procedures of Simulation}

We explain the functioning of our simulation model stepwise. Eight main steps sequentially divide the simulation process. The first and last steps bracket a loop that contains the core calculations. The operation of simulation can be classified into three blocks: consumer agent interaction among social network, e-commerce vendor agent, and advanced GAs which is used for calculating profit of vendor. The circle from step 2 to 7 is performed in an iterative manner until the exit threshold is reached. One circle 
repetition represents one simulation period. The start-to-end procedure process flowchart is represented in Figure 3.

Simulation starts with an initial configuration that populates with a set of 150 consumer Agents $C=\{1,2, \ldots, 150\}$ and one vendor Agent. We consider increase of consumer group with $1 \%$ increased rate. Draw upon Schramm' work, Consumer within a social network, are divided into three groups: innovators, early adopted and later adopted [16], each group has own acceptant rule to adopt the brand, and one agent can imitate adopted behavior of other neighbor agents. There are three consumers states in the consumer social network, the states include buying, democracy and non-shopping, at one time, each consumer has one state, the initial percentage is $0.4,0.3$, and 0.3 respectively. At time $t$, vendor make price, brand and technology strategy according to state of consumers at time $t-1$, and then consumer will perceived price level, brand image and technology strength of e-commerce vendor, also get information from WOM from other consumers, he/she make shopping decision, i.e., make state transition or change. System calculate profit of vendor based on consumers' state by using advanced GAs with 100 population size, and 0.5 mutation rate and 0.5 cross rate. System will run in an iterative mode until the exit condition is met.

In the simulation system, global and scenario constants are defined. Global constants remain unchanged across experiments. Table 1 shows a default vale of main parameters.

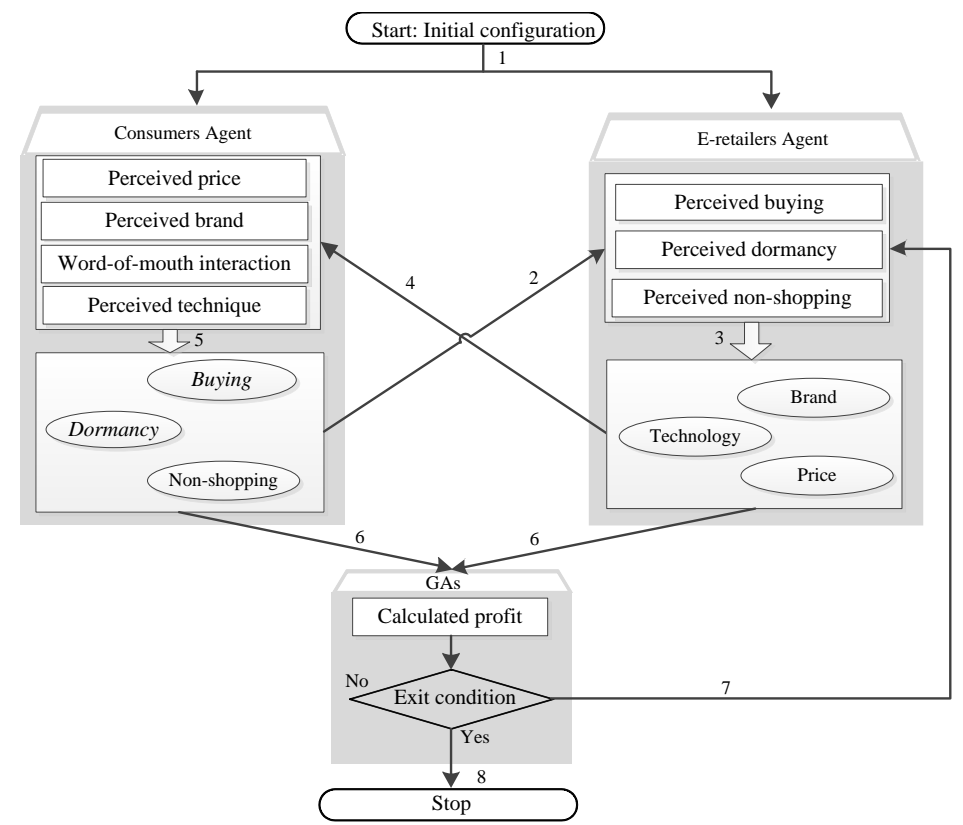

Figure 3. Flowchart of Simulation Model Procedures

Table 1. Default Values of Main Parameters in Experiment

\begin{tabular}{lllc}
\hline id & parameter & \multicolumn{1}{c}{ description } & default value \\
\hline 1 & num-nodes & number of nodes in network & 150 \\
2 & increased-nodes & probability of network nodes increase & 0.01 \\
3 & per-innovator & percentage of innovators & 0.3 \\
\hline
\end{tabular}


Table 1. Default Values of Main Parameters in Experiment

\begin{tabular}{lllc}
\hline id & \multicolumn{1}{c}{ parameter } & \multicolumn{1}{c}{ description } & default value \\
\hline 4 & per-earlyadopter & percentage of early adopters & 0.3 \\
5 & buying-init & percentage of buyer in initial network & 0.4 \\
6 & dormancy-init & Percentage of dormant buyer & 0.3 \\
7 & population-size & size of the whole population & 100 \\
8 & mutation-rate & percentage of mutated & 0.5 \\
9 & crossover-rate & percentage of crossovered & 0.5 \\
\hline
\end{tabular}

\subsection{Implementation}

NetLogo is a programmable modelling platform for natural and social science problems. It is established in 1999 by Uri Wilensky from the Center for Connected Learning (CCL) and Computer-Based Modeling; newer versions have been developed and published later on, the free Netlogo and some examples can be downloaded in the website as http://ccl.northwestern.edu/netlogo/. NetLogo 5.0 provides rich library of graphics, such as simulation and statistics graph, computational algorithms, etc, which are used in our implemented system. NetLogo is very suitable for modeling complex system which is a evolving system over time.

Based on NetLogo 5.0, we developed the experimental system. The main UI of our system is illustrated in Figure 4. There are three areas in the interface: on the left is where we can configure and command by pressing buttons; in the middle is the visually presented agent-based consumer network evolution; on the right are the curves of the trend of parameters.

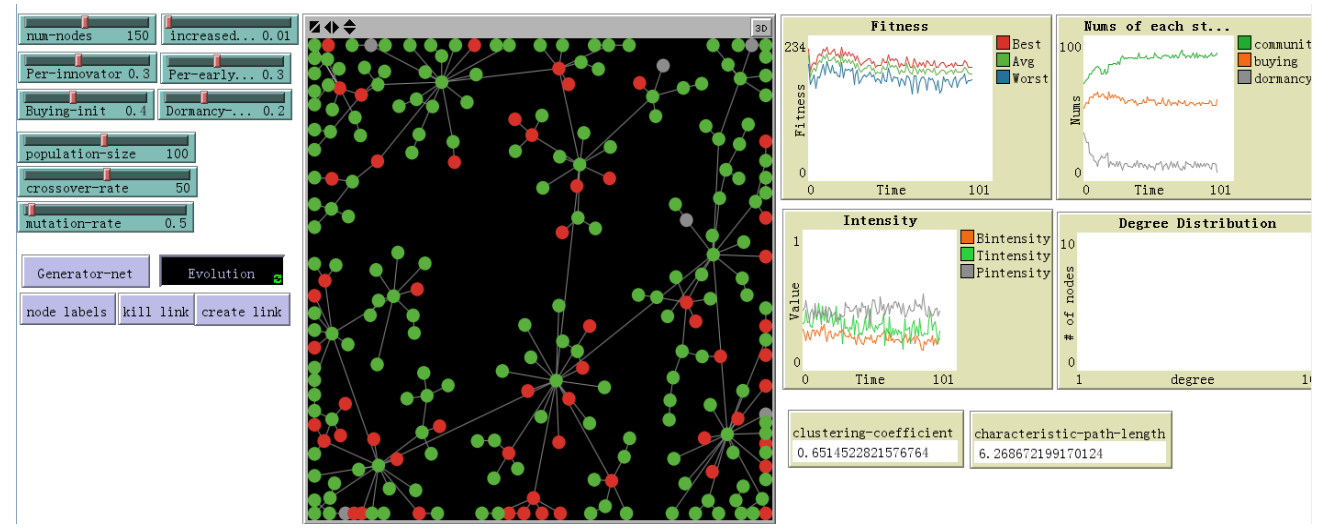

Figure 4. UI of the Experiment of the Co-evolution of Service Vendors and Consumer Behaviors

\subsection{Computational Experiment}

Experiments are carried out using default parameter values, and the evolution data are shown in Figure 5. 


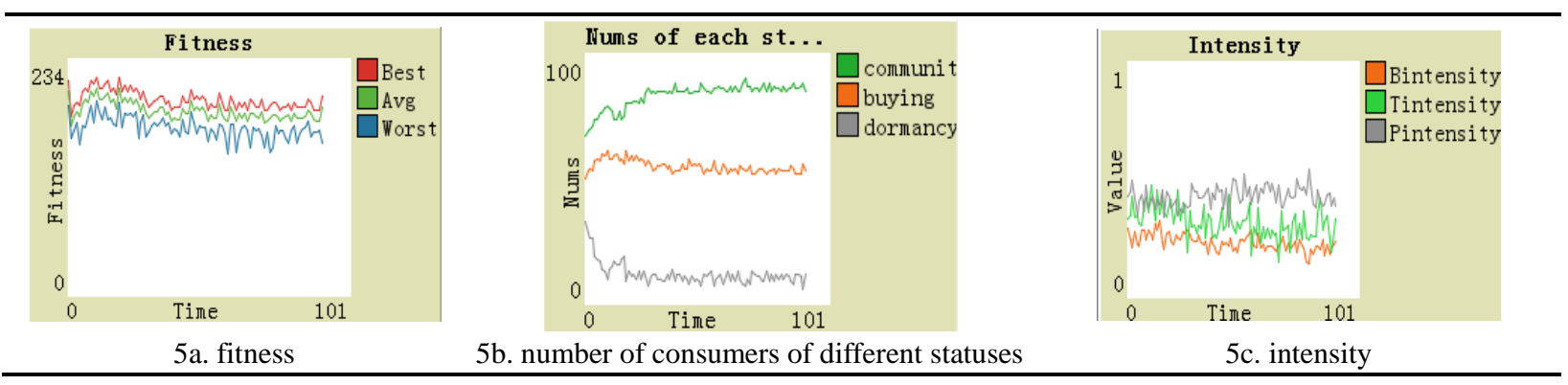

Figure 5. Evolution Curves

By analyzing the outputs of the experiment we find that service vendors' profits change over time, and the best fitness expectation is 193.54 while the worst is 165.88 . The curve of consumer status shows that potential buyers are growing, so the market management could be strengthened to bring out more customers. Buying consumers are going down then going up, consistent with traditional technique diffusion trend; dormant buyers are downward. In doing regression analysis in SPSS on buying consumers, we find that this curve fits very well with S-formula, and the regression formula is as fellow, $Y=e^{4.00419914488434-0.0219588906215591 x}$, and inverse formula, the regression formula is as fellow, $Y=e^{54.86959384764721 .03558774885386 / x}$, the result indicate that the trend of evolution is consistent with traditional technology diffusion, and thus the experiment can be used to make predictions in combination of those two regression formula in case.

The system can sample price, brand and ads intensity at different times to support decision making process. It can also collect data of the cluster coefficient and expected path length of network at different times; and these data can help analyze the effect of word of mouth among consumer network.

\section{Conclusion, Limitations and Further Research}

Computational experiment methods which integrated Agent, GA and complex network are used to study the co-evolution of e-commerce service vendors and consumers via a virtually reality system. The decision making process of e-commerce service vendors and consumers is described against the backdrop of collaborative e-commerce. Key algorithms such as consumer network generating algorithm, CA based adapted GA for service vendor decisions, etc, are designed for the co-evolution behaviors of both service vendors and customers. Computational experiment system is developed on NetLogo platform, and a virtual implemented sample is presented. The experiment system can provide technique support to e-commerce firms in decision making with regards to service providing, consumer behavior management, etc.

Although our simulation models can simulate dynamics of interaction between consumers and vendor, this study has several limitations that should be addressed in future research.

First, we only use classical theory validated our model. Although diffusion theory can properly explain adoption evolution, but I can't ensure evolution of profit be consistency with real systems. Future research will validate system with actual data.

Second, we present an agent-based model where consumers within each social network are rational, their decision model is symmetric. In reality, some consumers are not very rational, and so that their decision process may be on a regular basis that could also affect his/her adoption intention. Future research could examine rational and irrational types of consumers and their effect on adoption intention.

Finally, we have not do sensitive analysis of some of parameters. Further work will do more experiment to examine how parameter impact on evolution of vendor' profit and consumers' state, and then give more managerial and practical suggestions. 


\section{Acknowledgements}

This work was partially supported by a grant from the National Natural Science Foundation of China (No. 71101047).The first version of the work is represented in 2014 International Conference on Logistics, Informatics and Services Sciences (LISS'2014,Berkeley, California, USA).

\section{References}

[1] L. M. Camarinha-Matos, "Collaborative networked organizations - Concepts and practice in manufacturing enterprises", Computers \& Industrial Engineering, vol. 57, (2009), pp. 46-60.

[2] G. Jiang, "A review on the evolution of user acceptance behaviour in collaborative e-commerce", International Journal of Electronic Finance, vol. 6, (2012), pp. 62-78.

[3] M. A. Zaffar, "Diffusion dynamics of open source software: An agent-based computational economics (ACE) approach", Decision Support Systems, vol. 51, (2011), pp. 597-608.

[4] G. Jiang, "Agent-based Simulation of Competitive and Collaborative Mechanisms for Mobile Service Chains", Information Sciences, vol. 180, (2010) pp. 225-240.

[5] G. Jiang, "Agent-Based Simulation Approach to Understanding the Interaction between Employee Behavior and Dynamic Tasks", SIMULATION: Transactions of the Society for Modeling and Simulation International, vol. 87, (2011) pp. 407-422.

[6] M. E. J. Newman, "The structure and function of complex networks", SIAM Review, vol. 45, (2003), pp $167-256$.

[7] G. Jiang, "Evolution of knowledge sharing behavior in social commerce: An agent-based computational approach", Information Sciences, vol. 278, (2014), pp. 250-266.

[8] A.-L. Barabási and R. Albert, "Emergence of scaling in random networks", Science, vol. 286, (1999), pp. 509-512.

[9] R. A. Aliev, "Fuzzy-genetic approach to aggregate production-distribution planning in supply chain management", Information Sciences, vol. 177, (2007), pp. 4241-4255.

[10] J. F. Ardanuy and P. J. Zufiria, "Design and comparison of adaptive power system stabilizers based on neural fuzzy networks and genetic algorithms", Neurocomputing, vol. 70, (2007), pp. 2902-2912.

[11] P. Borisovsky, "Genetic algorithms for a supply management problem: MIP-recombination vs greedy decoder", European Journal of Operational Research, vol. 195, (2009), pp. 770-779.

[12] T. H. Hsu, "Selection of the optimum promotion mix by integrating a fuzzy linguistic decision model with genetic algorithms", Information Sciences, vol. 179, (2009), pp. 41-52.

[13] H. Iba, "Inference of differential equation models by genetic programming", Information Sciences, vol. 178, (2008), pp. 4453-4468.

[14] N. R. Sanders, "An empirical study of the impact of e-business technologies on organizational collaboration and performance", Journal of Operations Management, vol. 25, (2007), pp. 1332-1347.

[15] I. H. Toroslu and Y. Arslanoglu, "Genetic algorithm for the personnel assignment problem with multiple objectives", Information Sciences, vol. 177, (2007), pp. 787-803.

[16] M. E. Schramm, "An agent-based diffusion model with consumer and brand agents", Decision Support Systems, vol. 50, (2010), pp. 234-242.

\section{Author}

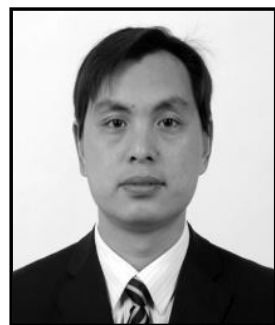

Guoyin Jiang, he is an associate Professor of Hubei University of Economics. He received his $\mathrm{PhD}$ from the Huazhong University of Science and Technology, China. His research interests include decision support systems, management System Simulation, and e-commerce. He has published over 40 professional papers in refereed journals and international conference proceedings. 